\title{
Contribution of Candida biomarkers and DNA detection for the diagnosis of invasive candidiasis in ICU patients with severe abdominal conditions
}

Cristóbal León ${ }^{1 *}$, Sergio Ruiz-Santana², Pedro Saavedra ${ }^{3}$, Carmen Castro ${ }^{4}$, Ana Loza ${ }^{1}$, Ismail Zakariya ${ }^{4}$, Alejandro Úbeda ${ }^{5}$, Manuel Parra ${ }^{4}$, Desirée Macías ${ }^{1}$, José Ignacio Tomás ${ }^{6}$, Antonio Rezusta ${ }^{7}$, Alejandro Rodríguez ${ }^{8}$, Frederic Gómez ${ }^{9}$, Estrella Martín-Mazuelos ${ }^{4}$ and The Cava Trem Study Group

\begin{abstract}
Background: To assess the performance of Candida albicans germ tube antibody (CAGTA), ( $\rightarrow$ 3)-B-D-glucan $(B D G)$, mannan antigen (mannan-Ag), anti-mannan antibodies (mannan-Ab), and Candida DNA for diagnosing invasive candidiasis (IC) in ICU patients with severe abdominal conditions (SAC).

Methods: A prospective study of 233 non-neutropenic patients with SAC on ICU admission and expected stay $\geq 7$ days. CAGTA (cutoff positivity $\geq 1 / 160$ ), BDG ( $\geq 80,100$ and $200 \mathrm{pg} / \mathrm{mL}$ ), mannan-Ag $(\geq 60 \mathrm{pg} / \mathrm{mL}$ ), mannan-Ab $(\geq 10 \mathrm{UA} / \mathrm{mL})$ were measured twice a week, and Candida DNA only in patients treated with systemic antifungals. IC diagnosis required positivities of two biomarkers in a single sample or positivities of any biomarker in two consecutive samples. Patients were classified as neither colonized nor infected $(n=48)$, Candida spp. colonization ( $n=154)$ (low-grade, $n=130$; high-grade, $n=24)$, and IC $(n=31)$ (intra-abdominal candidiasis, $n=20$; candidemia, $n=11$ ).

Results: The combination of CAGTA and BDG positivities in a single sample or at least one of the two biomarkers positive in two consecutive samples showed $90.3 \%$ (95\% Cl 74.2-98.0) sensitivity, $42.1 \%$ (95\% Cl 35.2-98.8) specificity, and 96.6\% (95\% Cl 90.5-98.8) negative predictive value. BDG positivities in two consecutive samples had $76.7 \%$ (95 \% Cl 57.7-90.1) sensitivity and 57.2 \% (95 \% Cl 49.9-64.3) specificity. Mannan-Ag, mannan-Ab, and Candida DNA individually or combined showed a low discriminating capacity.
\end{abstract}

Conclusions: Positive Candida albicans germ tube antibody and ( $\rightarrow 3$ )-B-D-glucan in a single blood sample or $(1 \rightarrow 3)$-B-D-glucan positivity in two consecutive blood samples allowed discriminating invasive candidiasis from Candida spp. colonization in critically ill patients with severe abdominal conditions. These findings may be helpful to tailor empirical antifungal therapy in this patient population.

Keywords: Invasive candidiasis, Candida spp. colonization, (1 $\rightarrow 3)$-B-D-glucan, Candida albicans germ tube antibody, Mannan antigen, Anti-mannan antibody, Candida PCR, Intra-abdominal candidiasis, Candidemia

\footnotetext{
* Correspondence: cleong@telefonica.net

Presented in part at the XVIII National Congress of the Spanish Society of Infectious Diseases, Sevilla, Spain, 29-31 May, 2015; L National Congress of the Spanish Society of Intensive, Critical and Coronary Care (SEMICYUC), San Sebastian, Spain, 14-17 June 2015; and the 7th Trends in Medical Mycology (TIMM), Lisbon, Portugal, 9-12 October, 2015.

${ }^{1}$ Intensive Care Unit, Hospital Universitario de Valme, Universidad de Sevilla, Avenida Bellavista s/n, 41014 Sevilla, Spain

Full list of author information is available at the end of the article
} 


\section{Background}

Accurate and timely diagnosis of invasive candidiasis (IC) from the patient's perspective and to optimize antifungal therapy in the intensive care unit (ICU) setting remains a topic of great interest [1-6]. The use of single or combined biomarkers, such as $(1 \rightarrow 3)-\beta$-D-glucan (BDG), Candida albicans germ tube antibody (CAGTA), mannan antigen (mannan-Ag), anti-mannan antibodies (mannan- $\mathrm{Ab}$ ), and polymerase chain reaction (PCR) detection of Candida DNA has received increasing attention [7-10], but the appropriate incorporation into clinical practice remains controversial. We investigated the performance of these five tests, alone and in combination, for discriminating IC in critically ill patients with severe abdominal conditions (SAC).

\section{Methods}

\section{Design and study population}

Between November 1, 2012 and February 28, 2014, all consecutive adult non-neutropenic patients with SAC on ICU admission and an expected stay of $\geq 7$ days were included in a prospective, cohort, observational, and multicenter study. The study protocol was approved by the Ethics Committee of Hospital Universitario de Valme (Sevilla, Spain) and the Spanish Agency for Medicines and Health Care Products (AEMPS). The codes and dates of approval of the study protocol were CEIC-A1, ref. 350/12 (May 29, 2012) for the Ethics Committee of Hospital de Valme, and September 14, 2012 for AEMPS. Informed consent was obtained from the patients or their legal representatives.

A severe abdominal condition (SAC) was defined as the process that caused gastrointestinal dysfunction or failure in the context of a medical abdominal disease (e.g., severe acute pancreatitis) or an abdominal surgical condition requiring elective or urgent procedures, with related complications (e.g., gastrointestinal perforation, hepatobiliary and pancreatic disorders, peritonitis, intraabdominal abscess, anastomotic leak, etc.) and prolonged postoperative stay after complicated abdominal surgery. Therefore, the definition of SAC included medical and surgical patients. Data for each patient was recorded using an electronic case report form.

Besides neutropenia defined as total leukocyte count $<$ $1000 / \mathrm{mm} 3$, other exclusion criteria on ICU admission were as follows: age below 18 years, human immunodeficiency virus (HIV) infection, active malignancy, current immunosuppression or immunosuppressive therapy, treatment with immunomodulating agents (monoclonal antibodies) in the previous 3 months, use of a dose of $\geq$ $20 \mathrm{mg} /$ day of prednisone or its equivalent within 1 month prior to ICU admission, spontaneous bacterial peritonitis in liver cirrhosis or advanced chronic liver disease, solid organ or bone marrow transplant recipients, chronic inflammatory bowel disease (ulcerative colitis, Crohn's disease), life expectancy less than 1 week, Acute Physiology and Chronic Health Evaluation (APACHE II) score $>35$ on ICU admission, documented Candida spp. infection during the week prior to ICU admission, treatment with antifungal agents before ICU admission or before inclusion in the study, limitation of the therapeutic effort, refusal to sign the informed consent, and inadequate data collection (incompleteness of the protocol specifications).

\section{Screening, microbiological cultures, and Candida score}

Surveillance cultures for the screening Candida spp. were performed twice a week from the fourth day of ICU admission. Surveillance samples were obtained from feces or rectal swabs, urine, tracheal aspirates (or protected specimen brush or bronchoalveolar lavage), oropharyngeal swabs (in patients without mechanical ventilation), peripheral blood, vascular lines, wound/ drainage exudates, or infected foci at the discretion of the attending physician. Samples were seeded directly into Candida CHROMagar ${ }^{\text {Tix }}$ Chromogen culture medium (Hardy Diagnostics, Santa Maria, CA, USA). All catheter tips removed were cultured in blood agar and Sabouraud agar by the Maki roll plate technique. Blood cultures were processed using the automated BACTEC ${ }^{\mathrm{max}}$ system (Becton Dickinson Diagnostic Instrument System, Paramus, NJ, USA) or other standardized methods. Results were considered positive in the presence of Candida growth in the culture medium. Identification at the species level was required. Candida score $[11,12]$ was calculated coinciding with collection of samples and once culture data were available.

\section{Serological biomarkers}

Blood samples $(15 \mathrm{~mL})$ were collected in three tubes without anticoagulant, centrifuged at $1800 \mathrm{rpm}$ for $10 \mathrm{~min}$, separated into aliquots, and stored at $-80{ }^{\circ} \mathrm{C}$ until analysis. None underwent more than one freezethaw cycle, and serum and reagents were tempered and homogenized before processing.

The BDG assay (Fungitell ${ }^{\mathrm{m}}$, Associates of Cape Cod, Inc., East Falmouth, MA, USA) was performed according to the manufacturer's recommendations: under laminar flow hood, duplicated and saving the mean value of both measurements and repeating the assay when between both BDG values there existed at least a $20 \%$ difference. Also, when the BDG values were greater than $500 \mathrm{pg} / \mathrm{mL}$, samples were diluted and retested. The cutoff value was $\geq 80 \mathrm{pg} / \mathrm{mL}$. CAGTA detection was performed by an immunofluorescence test (Vircell kit assay, Granada, Spain) according to the manufacturer's instructions. The cutoff value for positive CAGTA was $\geq 1 / 160$. For mannan-Ag and mannan-Ab, Platelia Candida Ag Plus and Platelia Candida Ab Plus were used in the 
automated EVOLISTM Twin Plus device (Bio-Rad, Marnes-la-Coquette, France). The cutoff values for positive mannan- $\mathrm{Ag}$ and mannan- $\mathrm{Ab}$ were $\geq 60 \mathrm{pg} / \mathrm{mL}$ and $\geq 10 \mathrm{AU} / \mathrm{mL}$, respectively. None of the tests were performed in real time and, therefore, were not available to clinician's decision making.

\section{Multiplex quantitative real-time PCR (MRT-PCR)}

The MRT-PCR assay [13] was performed to detect the six most frequent species of the genus Candida in invasive candidiasis (IC). The technique detected C. albicans, C. parapsilosis, C. tropicalis, C. glabrata, C. krusei, and C. guilliermondii using specific molecular beacon probes labeled with different fluorescent dyes: FAM, HEX, ROX and CYAN 500. Primers and probes were designed on the basis of the nucleotide sequences of the ITS ribosomal DNA region from strains belonging to the collection of the Spanish National Center of Microbiology. The probes targeted the ITS1 or ITS2 regions of ribosomal DNA. These regions were chosen as targets because of the possibility of designing a suitable probe for each case. Beacon Designer 5.0 software (Premier Biosoft, Palo Alto, CA, USA) was used to design primers and probes. The assay consisted of two multiplex PCRs: reaction one (C. albicans, C. parapsilosis, and C. tropicalis), which was performed using the LightCycler Probes Master Kit (Roche Diagnostics, Madrid, Spain); and reaction two (C. glabrata, C. krusei, and C. guilliermondii), which was performed using the $2 x$ Sensimix Probe Kit (Quantace, Ecogen, Madrid, Spain). Both PCRs were performed simultaneously in the LightCycler 480 System (Roche Diagnostics, Mannheim, Germany). DNA from blood and sera was extracted using the QIAamp DNA Mini Kit (Qiagen Izasa, Madrid, Spain). Elution was performed with $50 \mathrm{~mL}$ of buffer; the PCR was performed with $2 \mathrm{~mL}$ of DNA extracted from each sample. All samples were performed in duplicate and quantification standards were run in conjunction with each set of samples and negative controls.

\section{Study protocol and collection of data}

The following variables were recorded: age, sex, reason for ICU admission, APACHE II score, Sepsis-related Organ Failure Assessment (SOFA) score on ICU admission, comorbidities, and risk factors associated with Candida spp. colonization or infection. According to diagnosis at the time of ICU admission, patients were classified as medical or surgical. Surgical patients were those for whom the reason of ICU admission was the postoperative control of an elective or urgent surgical procedure. All surgical procedures and the number of operations performed in each patient were recorded. Medical patients undergoing major surgery during ICU admission were considered surgical patients.
Underlying diseases included diabetes mellitus treated with oral hypoglycemic agents and insulin-dependent diabetes, chronic obstructive pulmonary disease (COPD) (airflow limitation defined as forced expiratory volume during the first second $\left(\mathrm{FEV}_{1}\right) /$ forced vital capacity $(\mathrm{FVC})<88 \%$ predicted in men and $<89 \%$ predicted in women, or a postbronchodilator ratio of $\mathrm{FEV}_{1} / \mathrm{FVC}<0.7$ ), chronic liver disease (confirmed by liver biopsy or the presence of signs of portal hypertension, such as esophageal varices or ascites), chronic renal failure (in patients requiring hemodialysis or peritoneal dialysis), severe heart failure (defined as grades III and IV according to the New York Heart Association [NYHA] classification, alcoholism (defined as ethanol ingestion $>80 \mathrm{~g} /$ day), and HIV infection with adequate clinical control.

Risk factors for the development of Candida spp. colonization or infection included the following: central venous catheters, arterial catheters, total parenteral nutrition, mechanical ventilation, continuous renal replacement therapy (hemofiltration), treatment with corticosteroids (defined as intravenous administration of steroids for 5 days or more), and broad-spectrum antimicrobial treatment.

Once the patient was included in the study, the following data were recorded twice a week from the fourth day of ICU stay and for 3 weeks: surveillance cultures, Acute Physiology and Chronic Health Evaluation (APACHE II) score, Sepsis-related Organ Failure Assessment (SOFA) score, clinical situation assessment (presence or absence of sepsis, severe sepsis, or septic shock), and Candida score. Blood samples for the measurement of BDG, CAGTA, mannan-Ag, and mannan-Ab were drawn at the same time periods. In patients treated with empirical or directed systemic antifungal treatment (SAT), clinical and microbiological study controls were performed on days $0,+3,+7$, and +14 from the beginning of SAT. In addition, blood cultures and samples for PCR detection of Candida DNA were also obtained. Patients were followed until ICU and/or hospital discharge, or death. The indication of SAT was decided by the primary clinician. Details of SAT including starting date, drug(s), doses and duration of treatment were registered.

\section{Definitions}

Catheter-related candidemia was diagnosed in those patients who had an intravascular device and one or more positive cultures of blood samples obtained from the peripheral vein, clinical manifestations of infection (e.g., fever, chills, and/or hypertension), and no apparent source for bloodstream infection (with the exception of the catheter), as well as a positive catheter culture, either semiquantitative culture ( $\geq 15$ colony-forming units [cfu] per catheter segment) or quantitative $(\geq 1000 \mathrm{cfu}$ per catheter segment), whereby the same organism (species 
and susceptibility) were isolated from a catheter segment and a peripheral blood sample. Candiduria was defined in the presence of at least $10^{4} \mathrm{cfu} / \mathrm{mL}$ of the same Candida spp.

When BDG, CAGTA, mannan-Ag, and mannan-Ab were assessed independently, positivity for IC required values of any biomarker at or above the cutoff level in two consecutive samples. Candida PCR (C-PCR) was considered positive when Candida DNA was detected at least in a single sample. When the combination of two biomarkers was assessed, positivity for IC required values of both biomarkers at or above the cutoff level in a single sample, or values at or above the cutoff level for at least one of these biomarkers in two consecutive samples.
Candida colonization was considered unifocal when Candida spp. was isolated from one site and multifocal when Candida spp. was simultaneously isolated from various noncontiguous sites, even if two different Candida spp. were isolated. Low-grade Candida colonization was defined when Candida spp. was isolated in one or more foci, in one or two consecutive surveillance controls. High-grade Candida spp. colonization was defined as colonization of at least three body sites on two or more consecutive screening days [14]. Invasive candidiasis or proven Candida infection was defined as (i) primary candidemia (presence of Candida spp. in one or more blood cultures obtained from peripheral veins), and (ii) intra-abdominal candidiasis (IAC) (on the basis of macroscopic findings and direct examination or positive culture for Candida spp. of the peritoneal

\section{Methodology (details)}

\section{IC Diagnosis}

Clinical and microbiological controls

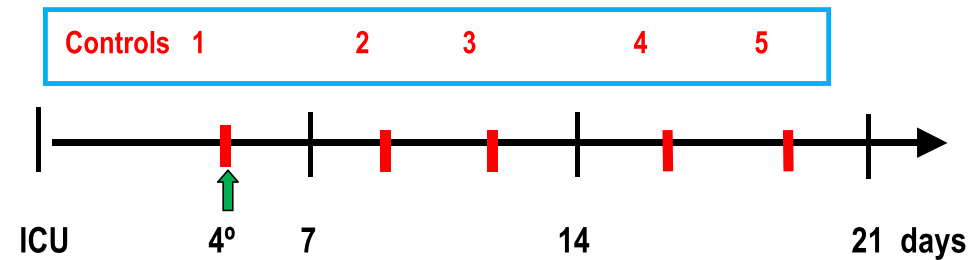

Controls: $4^{\circ}$ day after ICU admission and two weekly ( 5 total)

APACHE II / SOFA

Clinical status assessment.

Microbiological screening

Candida score.

Biomarkers determination

Systemic Antifungal therapy (SAT)

Clinical and microbiological controls

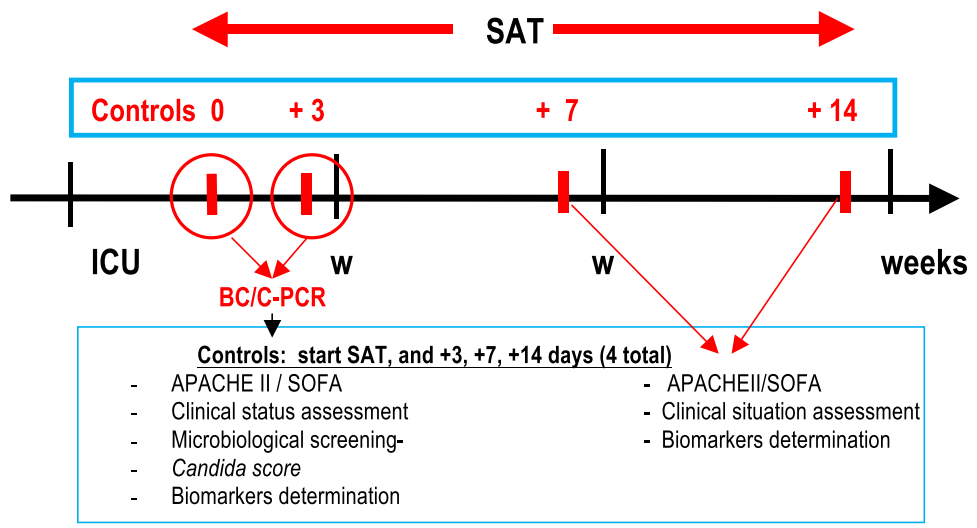

ICU: intensive care unit, APACHE II: Acute Physiology and Chronic Health Evaluation, SOFA: Sequential Organ Failure Assessment, BC: blood cultures, C-PCR: Candida PCR

Fig. 1 Details of the study methodology 
fluid collected during operation or within $24 \mathrm{~h}$ from external drainage) [15].

Patients were classified into the groups of neither colonized nor infected, low-grade Candida spp. colonization, high-grade Candida spp. colonization, and IC. In the presence of candidemia, catheters were removed, and fundoscopy was performed.

Clinical, microbiological, and serological controls performed during the diagnostic phase are shown in Fig. 1. When the patient received systemic antifungal therapy (SAT) (treatment phase), controls were performed at different time schedules and detection of Candida DNA by PCR assay was included in the two first determinations.

\section{Statistical analysis}

Categorical variables are expressed as frequencies and percentages, and continuous variables as mean and standard deviation (SD) when data followed a normal distribution, or as median and interquartile range (25th- 75th percentile) when distribution departed from normality. The percentages were compared using the chisquare $\left(x^{2}\right)$ test, the means by the $F$ test, and the medians by the Kruskal-Wallis test. Statistical significance was set at $P<0.05$. The performance of the BDG assay was analyzed with $\geq 100$ and $\geq 200 \mathrm{pg} / \mathrm{mL}$ cutoffs besides the cutoff of $\geq 80 \mathrm{pg} / \mathrm{mL}$. Sensitivity, specificity, and predictive values for the ability of each biomarker to discriminate between the IC and the remaining groups were calculated. Data were analyzed using the $\mathrm{R}$ package, version 3.1.0 ( $\mathrm{R}$ Development Core Team, 2014) [16].

\section{Results}

Study population and salient findings

The flow chart of the study population is shown in Fig. 2. Of 322 eligible patients, 89 (27.6 \%) were excluded for different reasons (Fig. 2). Data of 233 patients (67 \% men, mean age 66.7 years) were

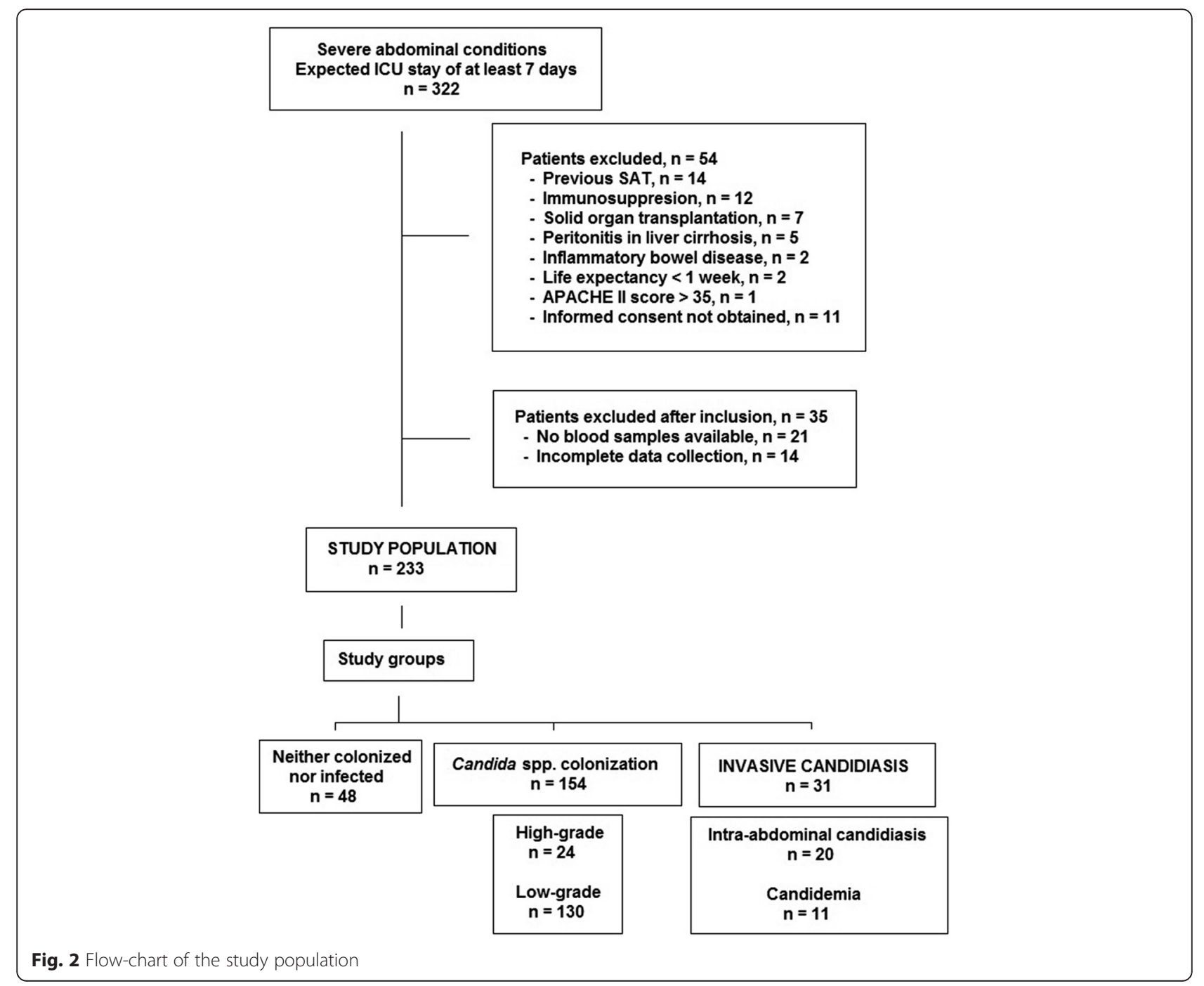


Table 1 Details of surgical procedures

Operation
Surgery of the esophagus
Esophagectomy with/without gastrectomy
Surgery of the stomach
Gastric bypass (gastroduodenostomy, gastrojejunal, Roux-en-Y)
Radical subtotal gastrectomy
Total gastrectomy
Partial gastrectomy plus vagus nerve transection
Gastrorraphy
Gastrostomy
Surgery of the gallbladder, biliary tract, and liver

$\frac{\text { Surgical procedures }}{1 \text { st } \quad \text { 2nd } \quad 3 r d}$

Cholecystectomy

Biliary tract surgery (cholecystostomy, bypasses, sphincteroplasty, etc.)

Segmental hepatectomy

7

7

1

Resection hydatid cyst

Open drainage of liver abscess

Resection hepatic tumor

Multiple or simple hepatorrhaphy

1

Surgery of the pancreas

Duodenocephalic pancreatectomy

Drainage pancreatic abscess

Distal, subtotal pancreatectomy or resection of pancreatic lesions

Surgery of the small intestine and colon

Intestinal resection: duodenectomy, enterocolectomy, enterectomy, jejunectomy

Subtotal colectomty (includes hemicolectomy and sigmoidectomy)

Colostomy or ileostomy (includes sigmoidostomy) duodenostomy

Intestinal anastomoses without resection (small intestine, small-large intestine, large intestine)

Total colectomy

Appendectomy

Closure of stoma

Drainage of diverticular abscess

Reduction of intestinal volvolus. Intestinal intussusception

Meckel's diverticulum resection

Retroperitoneum

Drainage of intraperitoneal abscess (including epiploic, iliac fossa, perisplenic and perigastric)

Enterotomy: foreign body

Drainage retroperitoneal abscess

Other

Exploratory laparotomy

Drainage of abdominal wall abscess

Hernia repair with/without mesh

Splenectomy. Splenorrhaphy

Drainage subphrenic or subdiaphragmatic abscess

24

$\begin{array}{lll}1 & 1 & 2\end{array}$


Table 1 Details of surgical procedures (Continued)

\begin{tabular}{lcc}
\hline Aortobifemoral bypass & 1 & 1 \\
Endovascular repair of abdominal aortic aneurysm & 1 & 1 \\
Placement Bogota bag & 1 & 1 \\
Nleofemoral thromboendarterectomy & 1 \\
& 426 \\
\hline
\end{tabular}

analyzed. In relation to SAC, 211 patients underwent 426 operations (66\% on an emergency basis; $50 \%$ related to the colon, biliary tract, and pancreas; $37 \%$ reoperations) and 22 had acute pancreatitis. Details of surgical procedures are shown in Table 1.

Thirty-one patients had IC (IAC 20; candidemia 11), 154 Candida spp. colonization (low-grade 130; highgrade 24), and 48 were neither colonized nor infected. The groups of IAC and high-grade colonization showed significant differences as compared to the remaining groups in Candida score, length of ICU stay, and number of surgical procedures; also, antifungal treatment was significantly more frequent among patients with IC (Table 2).

Data on risk factors, Candida colonization, $\mathrm{CI}$, and SAT are shown in Table 3. Twenty-three (74.1\%) of the 31 patients with IC had sepsis or septic shock, with a median time between ICU admission and diagnosis of infection of 7 days. The most common causative pathogens were C. albicans (51.6\%) and C. glabrata (22.6\%). Of the 31 patients with IC, 29 (93.5\%) received SAT (ICA 18, candidemia 11) and 2 died before indication of antifungal therapy. The median time between ICU admission and beginning of SAT was similar for patients with candidemia (6.5 days) and patients with IAC (7 days). Empirical SAT was administered in 90 patients (75.6\%), with severe sepsis or septic shock in 65, and a median time between ICU admission and starting treatment of 7 days. Patients treated with SAT for suspected or documented Candida spp. infection showed similar characteristics, except for a higher Candida score in patients with IC (median [IQR] 4 [3, 4] vs. 4 [3-5], $P=0.031)$.

Diagnostic value of BDG (cutoffs 80,100 and $200 \mathrm{pg} / \mathrm{mL}$ ), CAGTA, mannan-Ag, mannan-Ab, and C-PCR alone

BDG, CAGTA, mannan-Ag, and mannan-Ab were measured in 860 samples (3.6 per patient), and C-PCR in 213 , with positive results in 453, 306, 287, 150, and 110 samples, respectively. As shown in Tables 4 and 5, the percentages of patients with positive BDG, CAGTA, and mannan-Ag were significantly higher in the groups of candidemia, IAC, and high-grade Candida spp. colonization than in the remaining groups. When the BDG assay was used with the different cutoffs, the number of patients with positive results remained without changes in the group with candidemia but decreased in the groups of high-grade Candida spp. colonization and IAC. Positivity of the BDG test (cutoff $80 \mathrm{pg} / \mathrm{mL}$ ) showed the highest sensitivity $(76.7 \%)$ and negative predictive value $(94.1 \%)$ as compared to other assays, although CAGTA showed a higher specificity (64.3\%) and a lower sensitivity (53.3 \%) than BDG but the specificity of BDG improved when increasing the cutoff from $80 \mathrm{pg} / \mathrm{mL}$ to $200 \mathrm{pg} / \mathrm{mL}$. C-PCR had a sensitivity of $84 \%$ and a specificity of $32.9 \%$. All 20 C-PCR healthy controls were negative. The remaining tests showed a much lower reliability for the diagnosis of IC.

\section{Diagnostic value of combined use of BDG (cutoffs 80, 100 and $200 \mathrm{pg} / \mathrm{mL}$ ), CAGTA, mannan-Ag, mannan-Ab, and C-PCR}

Positivity of the BDG test for the three thresholds (or cutoffs) combined with positive results of the CAGTA, mannan-Ag, and mannan-Ab tests, as well as positivity of the CAGTA test combined with positive results of the mannan-Ag and mannan-Ab tests were significantly more frequent among patients with IAC, candidemia, and high-grade Candida spp. colonization than among those with low-grade Candida colonization and neither colonized nor infected (Table 6). Similar results were obtained for the combinations of positivities of C-PCR and mannan-Ag as well as mannan-Ag and mannan-Ab, which were also significantly more frequent among patients with IC and high-grade colonization.

As shown in Table 6, in the group of candidemia, the number of 10 patients with BDG and CAGTA positivities remained unchanged despite increasing the cutoff of BDG from $80 \mathrm{pg} / \mathrm{mL}$ to $200 \mathrm{pg} / \mathrm{mL}$. By contrast in the group of IAC, the number of patients with BDG and CAGTA positivities decreased from 18 at the cutoff of $80 \mathrm{pg} / \mathrm{mL}$ to 16 at $100 \mathrm{pg} / \mathrm{mL}$ and 14 at $200 \mathrm{pg} / \mathrm{mL}$. In the group of high-grade Candida spp. colonization, even increasing the cutoff from $80 \mathrm{pg} / \mathrm{mL}$ to $200 \mathrm{pg} / \mathrm{mL}, 19$ of the 24 patients continued to show positivities of both BDG and CAGTA biomarkers. When the group of neither colonized nor infected or with low-grade Candida spp. colonization was analyzed, the number of patients with positive BDG and positive CAGTA decreased from 
Table 2 Characteristics of the study population according to colonization and infection status

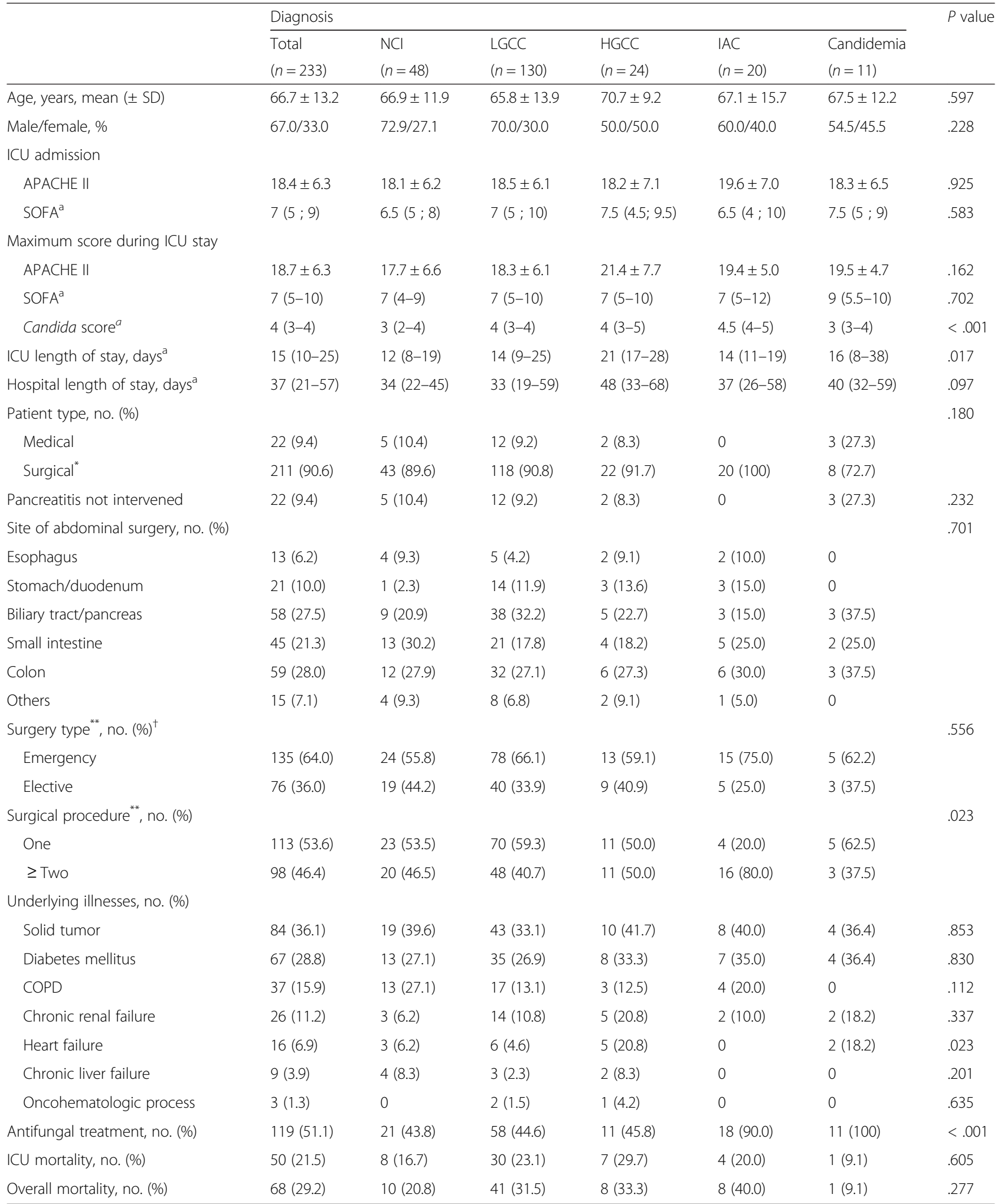

Values are expressed as frequencies and percentages; mean \pm standard deviation (SD); ${ }^{\text {amedian }}$ (25th-75th percentiles); $P$ value: statistical significance $\mathrm{NCl}$ neither colonized nor infected, LGCC low-grade Candida spp. colonization, HGCC high-grade Candida spp. colonization, IAC intra-abdominal candidiasis, ICU intensive care unit, APACHE II Acute Physiology and Chronic Health Evaluation, SOFA Sequential Organ Failure Assessment, COPD chronic obstructive pulmonary disease

*Abdominal surgery; ${ }^{* *} 211$ patients, ${ }^{\dagger}$ first surgical procedure 
Table 3 Risk factors, Candida colonization (low and high grade) and infection, and antifungal therapy characteristics of patients

\begin{tabular}{|c|c|}
\hline Included patients & $n=185^{*}$ \\
\hline \multicolumn{2}{|l|}{ Risk factors, no. (\%) } \\
\hline Broad-spectrum antibiotics & $184(99.5)$ \\
\hline Urinary catheter & $183(98.9)$ \\
\hline Central venous catheter & $183(98.9)$ \\
\hline Arterial catheter & $165(89.2)$ \\
\hline Mechanical ventilation & $156(84.3)$ \\
\hline Parenteral nutrition & $155(83.8)$ \\
\hline Corticosteroids & $73(39.5)$ \\
\hline Renal replacement therapy & $54(29.2)$ \\
\hline \multicolumn{2}{|l|}{ Candida colonization $(n=154)$} \\
\hline Low-grade Candida spp. colonization, no. (\%) & $130(84.4)$ \\
\hline High-grade Candida spp. colonization, no. (\%) & $24(15.6)$ \\
\hline Candida score $\geq 3$; first week/during study & $96 / 142(51.9)$ \\
\hline \multicolumn{2}{|l|}{ Candida infection $(n=31)$} \\
\hline \multicolumn{2}{|l|}{ Severity of Candida infection at diagnosis } \\
\hline No sepsis/sepsis & $3 / 5$ \\
\hline Severe sepsis/septic shock & $9 / 14$ \\
\hline \multicolumn{2}{|l|}{ Causative Candida spp } \\
\hline C. albicans & $16(51.6)$ \\
\hline C. glabrata & $7(22.6)$ \\
\hline C. parapsilosis & $3(9.7)$ \\
\hline C. tropicalis & $13.2)$ \\
\hline C. krusei & $1(3.2)$ \\
\hline C. dubliniensis & $1(3.2)$ \\
\hline C. famata & $1(3.2)$ \\
\hline C. albicans + C. glabrata & $1(3.2)$ \\
\hline Time between hospital admission to infection, days ${ }^{a}$ & $16(6.5-19.5)$ \\
\hline Time between ICU admission to infection, days ${ }^{a}$ & $7(2-13)$ \\
\hline Hospital/ICU mortality, no. (\%) & $9(29.0 / 5(16.1)$ \\
\hline \multicolumn{2}{|l|}{ Systemic antifungal therapy (SAT) $(n=119)^{* *}$} \\
\hline \multicolumn{2}{|l|}{ Empirical therapy for suspected IC $(n=90)^{* * *}$} \\
\hline Multifocal colonization, no. (\%) & $50(55.6)$ \\
\hline \multicolumn{2}{|l|}{ Clinical situation, no. (\%) } \\
\hline No sepsis/sepsis & $8(8.9) / 17(18.9)$ \\
\hline Severe sepsis/septic shock & $29(32.2) / 36(40.0)$ \\
\hline APACHE ॥ & $17.8 \pm 6.2$ \\
\hline SOFA ${ }^{a}$ & $6(5-9)$ \\
\hline Candida score & $4(3-4)$ \\
\hline $\begin{array}{l}\text { Time between of hospital/and beginning of SAT, } \\
\text { days }^{\mathrm{a}}\end{array}$ & $11(7-19)$ \\
\hline $\begin{array}{l}\text { Time between ICU admission and beginning of SAT, } \\
\text { days }^{\mathrm{a}}\end{array}$ & $7(1-11)$ \\
\hline Hospital/ICU mortality, no. (\%) & $29(32.2) / 23$ \\
\hline
\end{tabular}

Table 3 Risk factors, Candida colonization (low and high grade) and infection, and antifungal therapy characteristics of patients (Continued)

\begin{tabular}{ll}
\hline Therapy for documented infection $(n=29)^{* * *}$ & \\
Clinical situation & $3(10.3) / 4(13.8)$ \\
No sepsis/sepsis & $8(27.6) / 14(48.3)$ \\
Severe sepsis/septic shock & $18.8 \pm 6.8$ \\
APACHE II & $6.5(4-10)$ \\
SOFA & $4(3-5)$ \\
Candida score & \\
Time between of hospital/ICU admission and & \\
beginning of SAT, days ${ }^{\mathrm{a}}$ & $15(4.5 ; 22) / 6.5(1 ;$ \\
Candidemia (11) & $16)$ \\
& $12.5(8 ; 17) / 7(3 ; 12)$ \\
Intra-abdominal candidiasis (18) & $15(8-21)$ \\
Antifungal agents (>1 agent/patient) & \\
Days of therapy (first SAT) &
\end{tabular}

Values are expressed as frequencies and percentages; mean \pm standard

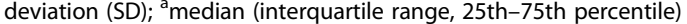

ICU intensive care unit, SAT systemic antifungal therapy, IC invasive candidiasis, APACHE II Acute Physiology and Chronic Health Evaluation, SOFA Sequential Organ Failure Assessment

"Patients with Candida colonization (low and high grade) $(n=154)$ plus invasive candidiasis $(n=31),{ }^{* *}$ patients with Candida colonization $(n=90)$ and IC $(n=29)$;

starting antifungal therapy

96 (53.9 \%) for a cutoff of $80 \mathrm{pg} / \mathrm{mL}$ to $70(39.3 \%)$ for a cutoff of $200 \mathrm{pg} / \mathrm{mL}$.

The combination of BDG positivity with positive values of other markers showed the highest sensitivities (Table 7). BDG (cutoff $80 \mathrm{pg} / \mathrm{mL}$ ) plus CAGTA showed a sensitivity of $90.3 \%$ and a negative predictive value of $96.6 \%$, although the specificity was only $42.1 \%$, increasing to $55.9 \%$ when the cutoff of BDG was $200 \mathrm{pg} / \mathrm{mL}$. The combination of BDG and mannan-Ag showed high sensitivities and negative predictive values $(80.6 \%$ and $93.7 \%$, respectively), but a low specificity (44.1\%). C-PCR showed the best specificities when combined with mannan- $\mathrm{Ag}$ and mannan- $\mathrm{Ab}(63.5 \%$ and $78.7 \%$, respectively). The remaining combinations were less relevant.

In the association of positive BDG and positive CAGTA, which was the combination with the highest diagnostic performance, the sensitivity decreased and the specific increased as the cutoffs changed from $80 \mathrm{pg} / \mathrm{mL}$ to $100 \mathrm{pg} / \mathrm{mL}$ and to $200 \mathrm{pg} / \mathrm{mL}$.

\section{Discussion}

Because of difficulties in the diagnosis IC in ICU settings, up to two thirds of critically ill patients with suspicion of fungal infection are given empirical antifungals $[17,18]$, a strategy with a well-recognized negative impact on adverse effects, risk of resistances [5], length of stay, patient's outcome, and health care costs $[19,20]$. In the present series, $51 \%$ of patients received SAT and 
Table 4 Number of patients with BDG (cutoff 80, 100 and 200 pg/mL), CAGTA, MANNAN biomarkers and Candida PCR positives used alone in the five study groups

\begin{tabular}{|c|c|c|c|c|c|c|}
\hline & \multicolumn{6}{|c|}{ Candida spp colonization } \\
\hline & $\overline{\mathrm{NCl}}$ & LGCC & $\mathrm{HGCC}$ & IAC & Candidemia & $P$ \\
\hline & $N=48$ & $N=130$ & $N=24$ & $N=20$ & $N=11$ & \\
\hline $\mathrm{BDG} \geq 80$ pg/mL, no. (\%) & $16 / 46(34.8)^{a}$ & $50 / 124(40.3)^{a}$ & $17 / 24(70.8)^{b}$ & $15 / 20(75.0)^{\mathrm{b}}$ & $8 / 10(80.0)^{b}$ & $<.001$ \\
\hline BDG $\geq 100$ pg/mL, no. (\%) & $16 / 46(34.8)^{\mathrm{a}}$ & $45 / 125(36.0)^{\mathrm{a}}$ & $14 / 24(58.3)^{a, b}$ & $13 / 20(65.0)^{\mathrm{b}}$ & $8 / 10(80.0)^{b}$ & .004 \\
\hline BDG $\geq 200$ pg/mL, no. (\%) & $10 / 47(21.3)^{a}$ & $20 / 128(15.6)^{a}$ & $11 / 24(45.8)^{b}$ & $10 / 20(50.0)^{b}$ & $8 / 10(80.0)^{b}$ & $<.001$ \\
\hline CAGTA positive, no. (\%) & $10 / 47(21.3)^{a}$ & $44 / 128(34.4)^{\mathrm{a}}$ & $17 / 24(70.8)^{b}$ & $8 / 20(40.0)^{a, b}$ & $8 / 10(80.0)^{b}$ & $<.001$ \\
\hline Mannan-Ag positive, no. (\%) & $10 / 48(20.8)^{a}$ & $40 / 127(31.5)^{\mathrm{a}}$ & $15 / 24(62.5)^{b}$ & $8 / 20(40.0)^{a, b}$ & $5 / 10(50.0)^{a, b}$ & .007 \\
\hline Mannan-Ab positive, no. (\%) & $6 / 48(12.5)$ & $12 / 128(9.4)$ & 4/24 (16.7) & $5 / 20(25.0)$ & $3 / 11(27.3)$ & .126 \\
\hline C-PCR positive, no. (\%) & $14 / 23(60.9)$ & $37 / 54(68.5)$ & $6 / 8(75.0)$ & $12 / 14(85.7)$ & 9/11(81.8) & .525 \\
\hline
\end{tabular}

Values are frequencies and percentages. Different superscripts $\left({ }^{a, b}\right)$ indicate nominally significant differences $(P<0.05)$. Biomarker positives: two determinations consecutives positives ( $\geq$ cutoff). C-PCR positive: one determination positive

$N C I$ neither colonized nor infected, $L G C C$ low-grade Candida spp. colonization, HGCC high-grade Candida spp. colonization, IAC intra-abdominal candidiasis, BDG (1-3)-B-D-glucan, CAGTA Candida albicans germ tube antibody, mannan-Ag mannan antigen, mannan-Ab mannan antibody, C-PCR PCR-based Candida detection

$75.6 \%$ of them did not have documented IC. Therefore, the availability of accurate tests to support either the initiation or discontinuation of SAT is a decision of crucial importance. The clinician can prescribe SAT if two consecutive [21-26] or more positive BDG determinations within 48 hours are present, or may stop SAT when BDG and CAGTA are negative in a single sample due to the high negative predictive value of this combination. Also, performance of BDG as a complementary test to blood cultures is currently under the scope of various clinical recommendations [27-29]. Bailly et al. [30] have recently demonstrated that in the absence of proven IC empiric SAT treatment could be safely stopped after 5 days of therapy without apparent deleterious effect on day-28 mortality.

According to the present findings, positive BDG and positive CAGTA levels in a single sample or at least positivity of one of the two biomarkers in two consecutive samples was the best way of characterizing IC diagnosis in non-neutropenic ICU patients with SAC. By contrast, individual or combined positivities of C-PCR, mannan-Ag, and mannan-Ab were of little value for discriminating IC from Candida spp. colonization. These results are relevant in daily practice for making decisions in complex clinical care settings.

Different studies have assessed the clinical value of CAGTA. The group of Martinez-Jiménez et al. [31] showed that the presence of a positive CAGTA test in a sample from a patient with candidemia suggests deepseated candidiasis, and more recently, provided evidence that the combination of CAGTA and BDG, or CAGTA and mannan-Ag, had high negative predictive values [32]. In 63 ICU patients and 37 non-ICU patients treated with antifungals, these authors achieved a sensitivity of $96.7 \%$ and negative predictive value of $97.1 \%$ when the combination (BDG and CAGTA at 1/60 cutoff) was positive on days $0,+3$, and +5 after starting antifungal therapy. In this study, serial determination of

Table 5 Performances of BDG (cutoff 80, 100 and 200 pg/mL), CAGTA, MANNAN biomarkers and C-PCR used alone for IC diagnosis

\begin{tabular}{lllll}
\hline Invasive candidiasis & $\begin{array}{l}\text { Sensitivity \% } \\
(95 \% \mathrm{Cl})\end{array}$ & $\begin{array}{l}\text { Specificity \% } \\
(95 \% \mathrm{Cl})\end{array}$ & $\begin{array}{l}\text { NPV \% } \\
(95 \% \mathrm{Cl})\end{array}$ & $\begin{array}{l}\text { PPV \% } \\
(95 \% \mathrm{Cl})\end{array}$ \\
\hline $\mathrm{BDG} \geq 80 \mathrm{pg} / \mathrm{mL}$ & $76.7(57.7-90.1)$ & $57.2(49.9-64.3)$ & $94.1(89.1-96.8)$ & $21.7(17.7-26.4)$ \\
$\mathrm{BDG} \geq 100 \mathrm{pg} / \mathrm{mL}$ & $70.0(50.6-85.3)$ & $61.5(54.3-68.4)$ & $93.0(88.4-95.9)$ & $21.9(17.3-27.3)$ \\
$\mathrm{BDG} \geq 200 \mathrm{pg} / \mathrm{mL}$ & $60.0(40.6-77.3)$ & $79.4(73.1-84.8)$ & $92.9(89.4-95.4)$ & $30.5(22.7-39.6)$ \\
CAGTA positive & $53.3(34.3-71.7)$ & $64.3(57.2-71.0)$ & $90.1(86.0-93.2)$ & $18.4(13.3-24.8)$ \\
Mannan-Ag positive & $43.3(25.5-62.6)$ & $67.3(60.3-73.8)$ & $88.7(85.0-91.6)$ & $16.7(11.3-24.0)$ \\
Mannan-Ab positive & $25.8(11.9-44.6)$ & $89.0(83.8-93.0)$ & $88.6(86.2-90.6)$ & $26.7(15.1-42.6)$ \\
C-PCR positive & $84.0(63.9-95.5)$ & $32.9(23.1-44.0)$ & $87.5(73.1-94.8)$ & $26.9(22.7-31.6)$ \\
\hline
\end{tabular}

Values are frequencies and percentages. Biomarker positives: two determinations consecutives positives ( $\geq$ cutoff). C-PCR positive: one determination positive $\mathrm{NCl}$ neither colonized nor infected, LGCC low-grade Candida spp. colonization, HGCC high-grade Candida spp. colonization, IAC intra-abdominal candidiasis, BDG (1-3)-ß-D-glucan, CAGTA Candida albicans germ tube antibody, mannan-Ag mannan antigen; mannan-Ab mannan antibody, C-PCR PCR-based Candida detection 
Table 6 Number of patients with BDG (cutoff 80, 100 and 200 pg/mL) CAGTA, MANNAN biomarkers and Candida PCR positives used combined in the five study groups

\begin{tabular}{|c|c|c|c|c|c|c|c|}
\hline & & \multicolumn{5}{|c|}{ Candida spp. colonization } & \multirow{3}{*}{$P$} \\
\hline & & $\mathrm{NCl}$ & LGCC & HGCC & IAC & Candidemia & \\
\hline & & $N=48$ & $N=130$ & $N=24$ & $N=20$ & $N=11$ & \\
\hline \multirow[t]{4}{*}{$\mathrm{BDG} \geq 80 \mathrm{pg} / \mathrm{mL}$} & CAGTA & $21(43.8)^{a}$ & $75(57.7)^{\mathrm{a}}$ & $21(87.5)^{b}$ & $18(90.0)^{b}$ & $10(90.9)^{b}$ & $<.001$ \\
\hline & Mannan-Ag & $21(43.8)^{a}$ & $71(54.6)^{\mathrm{a}, \mathrm{c}}$ & $21(87.5)^{b}$ & $16(80.0)^{b}$ & $9(81.8)^{b, c}$ & $<.001$ \\
\hline & Mannan-Ab & $21(43.8)^{a}$ & $61(46.9)^{a}$ & $19(79.2)^{\mathrm{b}, \mathrm{c}}$ & $15(75.0)^{c}$ & $8(72.7)^{a, c}$ & .002 \\
\hline & $C-P C R$ & $14 / 23(60.9)$ & $32 / 54(59.3)$ & $5 / 8(62.5)$ & 11/14 (78.6) & $8 / 11(72.7)$ & .724 \\
\hline \multirow[t]{4}{*}{$\mathrm{BDG} \geq 100 \mathrm{pg} / \mathrm{mL}$} & CAGTA & $21(43.8)^{a}$ & $72(55.4)^{a}$ & $20(83.3)^{b}$ & $16(80.0)^{b}$ & $10(90.9)^{b}$ & $<.001$ \\
\hline & Mannan-Ag & $21(43.8)^{a}$ & $68(52.3)^{a_{1} c}$ & $19(79.2)^{b}$ & $14(70.0)^{\mathrm{a}, \mathrm{b}, \mathrm{c}}$ & $9(81.8)^{c}$ & .009 \\
\hline & Mannan-Ab & $21(43.8)$ & $57(43.8)$ & $16(66.7)$ & $13(65.0)$ & $8(72.7)$ & .053 \\
\hline & C-PCR & $14 / 23(60.9)$ & 28/54 (51.9) & $5 / 8(62.5)$ & 10/14 (71.4) & $8 / 11(72.7)$ & .606 \\
\hline \multirow[t]{4}{*}{$\mathrm{BDG} \geq 200 \mathrm{pg} / \mathrm{mL}$} & CAGTA & $16(33.3)^{a}$ & $54(41.5)^{\mathrm{a}}$ & $19(79.2)^{\mathrm{b}}$ & $14(70.0)^{b}$ & $10(90.9)^{b}$ & $<.001$ \\
\hline & Mannan-Ag & $15(31.2)^{a}$ & $52(40.0)^{\mathrm{a}}$ & $18(75.0)^{b}$ & $13(65.0)^{b}$ & $9(81.8)^{b}$ & $<.001$ \\
\hline & Mannan-Ab & $13(27.1)^{\mathrm{a}}$ & $33(25.4)^{\mathrm{a}}$ & $13(54.2)^{b}$ & $12(60.0)^{b}$ & $8(72.7)^{b}$ & $<.001$ \\
\hline & C-PCR & $10 / 23(43.5)$ & 21/54 (38.9) & $3 / 8(37.5)$ & 10/14 (71.4) & $8 / 11(72.7)$ & .089 \\
\hline \multirow[t]{3}{*}{ CAGTA positive } & Mannan-Ag & $15(31.2)^{a}$ & $63(48.5)^{b}$ & $21(87.5)^{c}$ & $11(55.0)^{a, b}$ & $10(90.9)^{c}$ & $<.001$ \\
\hline & Mannan-Ab & $14(29.2)^{\mathrm{a}}$ & $53(40.8)^{a, c}$ & $18(75.0)^{b}$ & $12(60.0)^{b, c}$ & $8(72.7)^{b}$ & $<.001$ \\
\hline & $C-P C R$ & 8/23 (34.8) & $25 / 54(46.3)$ & $5 / 8(62.5)$ & $6 / 14(42.9)$ & $8 / 11(72.7)$ & .278 \\
\hline \multirow[t]{2}{*}{ C-PCR positive } & Mannan-Ag & $5 / 23(21.7)^{a}$ & $21 / 54(38.9)^{a}$ & $5 / 8(62.5)^{a, b}$ & $7 / 14(50.0)^{a}$ & $8 / 11(72.7)^{b}$ & .037 \\
\hline & Mannan-Ab & 8/23 (34.8) & 19/54 (35.2) & $1 / 8(12.5)$ & $7 / 14(50.0)$ & $8 / 11(72.7)$ & .073 \\
\hline Mannan-Ag positive & Mannan-Ab & $13(27.1)^{a}$ & $51(39.2)^{a, b}$ & $17(70.8)^{c}$ & $12(60.0)^{b, c}$ & $5(45.5)^{a, c}$ & .003 \\
\hline
\end{tabular}

Values are frequencies and percentages. Combined biomarkers and C-PCR: two consecutive determinations positives or/and at least one of each biomarkers (in one determination) positive. C-PCR: one determination positive

$\mathrm{NCI}$ neither colonized nor infected, LGCC low-grade Candida spp. colonization, HGCC high-grade Candida spp. colonization, IAC intra-abdominal candidiasis, BDG (1-3)-B-D-glucan, CAGTA Candida albicans germ tube antibody, mannan-Ag mannan antigen, mannan-Ab mannan antibody, C-PCR PCR-based Candida detection Different superscripts $(a, b, c)$ indicate nominally significant differences $(P<.05)$

BDG and CAGTA could be used to stop safely antifungals in $31 \%$ of patients receiving empirical antifungal therapy both in ICU and non-ICU wards [33].

The advantage of combining BDG and CAGTA for accurate diagnosis of IC is consistent with our findings in selected ICU patients with SAC, with a sensitivity of $90.3 \%$ and a negative predictive value of $96.6 \%$. Although the specificity was only $42.1 \%$, the combination of BDG and CAGTA was positive in 21 of 24 patients with high-grade Candida spp. colonization, which probably indicates a hidden IC or a high probability of developing candidemia, two circumstances in which starting empirical antifungals would be justified. Also, the high negative predictive value could be useful for excluding Candida spp. infection in patients receiving empirical SAT.

The combination of mannan-Ag and mannan- $\mathrm{Ab}$ has been also recommended [28, 34] but results of clinical studies are not very encouraging, with a diagnostic specificity of $51 \%$ and sensitivity of $77 \%$ in a retrospective analysis of 162 patients of whom 91 had proven IC [35]. In samples from 31 patients with candidemia and 50 patients with bacteremia, the use of mannan-Ag and mannan- $\mathrm{Ab}$ alone showed sensitivities of $64.3 \%$ and $61.5 \%$, specificities of $95.7 \%$ and $65.8 \%$, and negative predictive values of $81.8 \%$ and $71.4 \%$, respectively [32]. In our study, positive mannan-Ag tests were more frequent in patients with high-grade Candida colonization (65.5\%) and candidemia (50\%), whereas the rate of positive mannan-Ab tests was low in all study groups. However, both tests had a low sensitivity for diagnosing IC possibly in relation to the limited capacity to produce specific antibodies in the presence of immunosuppression $[9,33]$.

Currently, molecular techniques are increasingly used to diagnose invasive fungal infections $[8,9]$. Results of a meta-analysis with 54 studies and 4694 patients, PCR positivity rates among patients with proven or probable IC were $85 \%$, while blood cultures were positive for $38 \%$ [36]. In 63 ICU patients with suspected IC (eventually confirmed in 27) and 40 healthy controls, the sensitivity, specificity, positive predictive value and negative predictive value of MRT-PCR for the diagnosis of IC were $96.3 \%$, $97.3 \%, 92.8 \%$ and $98.7 \%$, respectively. 
Table 7 Performances of BDG (cutoff 80, 100 and 200 pg/mL), CAGTA, MANNAN biomarkers and C-PCR used combined for IC diagnosis

\begin{tabular}{|c|c|c|c|c|c|}
\hline \multicolumn{2}{|l|}{ Invasive candidiasis } & $\begin{array}{l}\text { Sensitivity \% } \\
(95 \% \mathrm{Cl})\end{array}$ & $\begin{array}{l}\text { Specificity \% } \\
(95 \% \text { Cl) }\end{array}$ & $\begin{array}{l}\text { NPV \% } \\
(95 \% \text { Cl) }\end{array}$ & \multirow{2}{*}{$\begin{array}{l}\text { PPV \% } \\
(95 \% \text { Cl) }\end{array}$} \\
\hline \multirow{5}{*}{$\mathrm{BDG} \geq 80 \mathrm{pg} / \mathrm{mL}$} & & & & & \\
\hline & काजि & 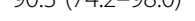 & $42.1(30.2-49.2)$ & $90.0(90.5-90.0)$ & $19.3(10.9-22.0)$ \\
\hline & Mannan-Ag & $80.6(62.5-92.5)$ & $44.1(37.1-51.2)$ & 93.7 (87.7-96.9) & $18.1(15.2-21.5)$ \\
\hline & Mannan-Ab & $74.2(42.9-57.1)$ & $50.0(42.9-57.1)$ & $92.7(87.2-95.9)$ & $18.5(15.1-22.6)$ \\
\hline & $C-P C R$ & 76.0 (54.9-90.6) & $40.0(29.5-51.2)$ & $85.0(72.9-92.3)$ & $27.1(22.0-33.0)$ \\
\hline \multirow[t]{4}{*}{$\mathrm{BDG} \geq 100 \mathrm{pg} / \mathrm{mL}$} & CAGTA & $83.9(66.3-94.5)$ & $44.1(37.1-51.2)$ & 94.7 (88.7-97.6) & 18.7 (15.9-21.9) \\
\hline & Mannan-Ag & $74.2(55.4-88.1)$ & $46.5(39.5-53.7)$ & $92.2(86.4-95.6)$ & $17.6(14.3-21.4)$ \\
\hline & Mannan-Ab & $67.7(48.6-83.3)$ & $53.5(46.3-60.5)$ & $91.5(86.5-94.8)$ & $18.3(14.4-22.9)$ \\
\hline & $C-P C R$ & $72.0(50.6-87.9)$ & $44.7(33.9-55.9)$ & $84.4(73.5-91.4)$ & $27.7(21.9-34.3)$ \\
\hline \multirow[t]{4}{*}{$\mathrm{BDG} \geq 200 \mathrm{pg} / \mathrm{mL}$} & CAGTA & 77.4 (58.9-96.9) & $55.9(48.8-62.9)$ & $94.2(89.3-96.9)$ & $21.2(17.4-25.6)$ \\
\hline & Mannan-Ag & $71.0(52.0-85.8)$ & $57.9(50.8-64.8)$ & $92.9(88.1-95.8)$ & $20.6(16.4-25.5)$ \\
\hline & Mannan-Ab & $64.5(45.4-80.8)$ & $70.8(64.0-77.0)$ & 92.9 (88.9-95.5) & $25.3(19.5-32.2)$ \\
\hline & $C-P C R$ & $72.0(50.6-87.9)$ & $60.0(48.8-70.5)$ & $87.9(79.1-93.3)$ & $34.6(27.0-43.1)$ \\
\hline \multirow[t]{3}{*}{ CAGTA positive } & Mannan-Ag & $67.7(48.6-83.3)$ & $51.0(43.9-58.1)$ & $91.2(85.9-94.6)$ & $17.5(13.8-21.9)$ \\
\hline & Mannan-Ab & $64.5(45.4-80.8)$ & $57.9(50.8-64.8)$ & $91.4(86.7-94.5)$ & $19.0(14.8-24.2)$ \\
\hline & $C-P C R$ & $56.0(34.9-75.6)$ & $55.3(44.1-66.1)$ & $81.0(72.5-87.4)$ & $26.9(19.5-35.9)$ \\
\hline \multirow[t]{2}{*}{ C-PCR positive } & Mannan-Ag & $60.0(38.7-78.9)$ & $63.5(52.4-73.7)$ & $84.4(76.5-90.0)$ & $32.6(24.0-42.5)$ \\
\hline & Mannan-Ab & $54.8(36.0-72.7)$ & $78.7(72.4-84.1)$ & 91.9 (88.4-94.4) & $28.3(20.7-37.5)$ \\
\hline Mannan-Ag positive & Mannan-Ab & $54.8(36.0-72.7)$ & $59.9(52.8-66.7)$ & 89.6 (85.2-92.8) & $17.3(12.8-23.1)$ \\
\hline
\end{tabular}

Also, in deep-seated IC, the sensitivity of PCR was $90.9 \%$ vs. $45.4 \%$ for blood cultures $(P=0.06)$ [13]. In our study, positive PCR tests were more frequent in patients with IC and high-grade Candida colonization, although the diagnostic accuracy was low. However, wide standardization of molecular tests has not been reached and results should be considered with caution.

Other studies have examined the combinations of biomarkers with inconsistent results. Nguyen et al. [37] in blood samples collected prospectively from 55 patients with IC and 73 controls, PCR and BDG were similar for diagnosing candidemia (59 \% vs. $68 \%$ ) but PCR was more sensitive for deep-seated candidiasis (89 \% vs. $53 \% ; P=0.004)$, and both (PCR and BDG) were more sensitive than blood cultures among patients with deepseated candidiasis. Held et al. [38] evaluated the usefulness of BDG, mannan-Ag with/without mannan-Ab, and Cand-Tec Candida antigen in a case-control study. The combination of BDG and mannan-Ag was superior to the other combinations with $89.3 \%$ sensitivity and $85.0 \%$ specificity. In a previous study of our group in ICU patients with SAC [20], BDG levels $>259 \mathrm{pg} / \mathrm{mL}$ combined with CAGTA positive results accurately discriminated Candida spp. colonization from IC (sensitivity $90.3 \%$, specificity $54.8 \%$, and negative predictive value $93.9 \%$ ).
This study has also its limitations. First, the number of IC was small and this is important because we think that BDG and CAGTA behave differently in candidemia and in deep-seated candidiasis, such as IAC. The number of patients was not sufficient for a reliable analysis of the differences in biomarkers accuracy between IAC and candidemia. Second, although we applied strict criteria for the diagnose IAC, Candida spp. isolation in peritoneal exudates is difficult and the role of unregistered polymicrobial cultures is hard to establish. Third, the BDG and PCR tests were performed in frozen samples, so that false negative results are possible due to instability of the samples. Fourth, the presence of intestinal mucositis may facilitate the translocation of Candida spp. through the gastrointestinal barrier and eventually may interfere with BDG measurements. Finally, we should take into account the potential impact of echinocandins on BDG synthesis because, in the current study, 119 patients, approximately half of the studied patients, received SAT and nearly $60 \%$ of them were treated with echinocandins.

Our study demonstrates that BDG, in contrast to other methods, is the best biomarker to be used in the diagnosis of IC in the critically ill patient with SAC. When BDG is used in combination with CAGTA, its diagnostic 
performance increases notably. The stratification of colonized patients (in high-grade and low-grade) and of IC (in candidemia and IAC) allowed us to observe that behavior of these biomarkers is different in these clinical scenarios, particularly when BDG with different cutoffs is used. The association of BDG and CAGTA positivities is an excellent diagnostic tool for candidemia, independently of the cutoff value of $B D G$, but this was not the case for IAC where the number of patients with BDG and CAGTA positivities decreases as the cutoff value of BDG increases. In cases of high-grade Candida spp. colonization, the percentage of patients with both BDG and CAGTA positive results decreases slightly (two patients) when the BDG cutoff increases, which can raise the possibility of a very likely presence of "occult" IC (especially candidemia). From a practical clinical point of view, it may be stated that in critically ill patients with SAC and BDG/CAGTA positivity, with a cutoff of BDG of $\geq 80 \mathrm{pg} / \mathrm{mL}$, the need to start antifungal treatment should be considered, covering the three aforementioned possible clinical scenarios. Another practical issue of combined BDG and CAGTA positivity is the high negative predictive value $(96.6 \%)$, which would raise the possibility of stopping an empirical antifungal therapy. However, in patients without Candida colonization or with low-grade Candida spp. colonization, the association of BDG and CAGTA is positive in $53.9 \%$ of patients, which logically decreases to $39.3 \%$ when a BDG cutoff of $200 \mathrm{pg} / \mathrm{mL}$ is used.

\section{Conclusions}

In selected, non-neutropenic critically ill patients with SAC, the combination of BDG and CAGTA positivities in a single determination or at least one of the two biomarkers positive in two consecutive samples, allowed discriminating between IC and the groups of low-grade and high-grade Candida colonization as well as neither colonized nor infected. Other tests including PCR Candida DNA detection and serum levels of mannan$\mathrm{Ag}$ and mannan- $\mathrm{Ab}$ alone or combined did not improve the diagnostic yield. These clinically relevant findings can be exploited to tailor empirical systemic antifungal therapy in ICU patients with SAC.

\footnotetext{
Abbreviations

Ab: antibody; AEMPS: Spanish Agency for Medicines and Health Care Products; Ag: antigen; APACHE II: Acute Physiology and Chronic Health Evaluation; BDG: $1 \rightarrow 3$ )-B-D-glucan; CAGTA: Candida albicans germ tube antibody; C-PCR: Candida polymerase chain reaction; FEV: forced expiratory volume; FVC: forced vital capacity; HIV: human immunodeficiency virus; IAC: intra-abdominal candidiasis; IC: invasive candidiasis; ICU: intensive care unit; MRT-PCR: multiplex quantitative real-time polymerase chain reaction; PCR: polymerase chain reaction; SAC: severe abdominal conditions; SAT: systemic antifungal therapy; SOFA: Sepsis-related Organ Failure Assessment.
}

\section{Competing interests}

Cristobal León has received research grants and consultant's honoraria from Astellas Pharma.
Sergio Ruiz-Santana has received educational grants and/or speaker's honoraria from Astellas Pharma.

Ana Loza has received educational grants and/or speaker's honoraria from Astellas Pharma.

There are no conflicts of interest for the remaining authors.

\section{Authors' contributions}

$\mathrm{CL}$ contributed to study design, writing of the study protocol and submission to the Institutional Review Board, review of individual patients' case report forms, data analysis and interpretation, results interpretation, literature search, writing of the manuscript, and approval of the final version. SR-S contributed to study design, data analysis and interpretation, writing of the manuscript with important contributions for scientific content, and approval of the final draft. PS contributed to statistical analysis and interpretation of data, writing of some parts of the manuscript, critical review, and approval of the final draft. CC contributed to laboratory analyses and interpretation of the results, data collection, review of the manuscript, and approval of the final draft. $A L, J I T, A R e, A R o, F G$, and DM contributed to screening, inclusion of the patients in the study and follow-up assessments, data collection, care of the patients, critical review of the manuscript, and approval of the final draft. IZ and MP contributed to laboratory analyses, data collection, review of the manuscript, and approval of the final draft. AÚ contributed to supervision and coordination of the online registries, review of the manuscript, and approval of the final draft. EM-M contributed to writing of research grants, data analysis and interpretation, literature search, writing of the manuscript, and approval of the final draft.

\section{Acknowledgements}

We thank Drs. Manuel Cuenca Estrella and María José Buitrago from the National Center of Microbiology (Madrid, Spain) for their invaluable active contribution in training us in the performance of the multiplex real-time PCR assay, and Marta Pulido, MD, for editing the manuscript and editorial assistance. The fees for medical editing were paid by the Fundación Pública Andaluza para la Gestión de la Investigación en Salud de Sevilla (FISEVI), Sevilla, Spain.

\section{Financial support}

This work was supported by a research grant (FIS PI 13/01168) from Instituto de Salud Carlos III, Madrid, (Spain) and Astellas Pharma. The funding body and Astellas Pharma had no role in study design and conduct, patient recruitment, data collection, analysis, and interpretation, writing of the manuscript, or decision to submit the manuscript for publication. This study had the scientific support of the Working Group of Infectious Diseases of the Society of Intensive, Critical Medicine and Coronary Units (SEMICYUC), the Medical Mycology Study Group of the Spanish Society of Infectious Diseases and Clinical Microbiology (GEMICOMED-SEIMC), and the Spanish Mycology Association (AEM).

\section{The Cava Trem Study Group}

J.I. Tomás, M. Gurpegui, V. González, and A. Rezusta (Hospital Universitario Miguel Servet, Zaragoza); C. León, E. Martin-Mazuelos, C. Castro, A. Loza, A. Úbeda, M. Parra, I. Zakariya, and D. Macías (Hospital Universitario de Valme, Sevilla); A. Úbeda (Hospital Punta de Europa, Algeciras, Cádiz); A. Rodríguez, M. Bodi, F. Gómez, S. Trefler, M. Llauradó, and R. Güell (Hospital Universitari Joan XXIII, Tarragona); R. González, F. J. Díaz, Raúl González, J.M. Marcos, D. Carriedo, I. Fernández, E. Valverde, A. Esteban, and T. Marrodan (Complejo Asistencial de León, León); A. Arenzana, C. González, A. I. Suárez (Hospital Universitario Virgen Macarena, Sevilla); J. Ballús, F. Esteve, and J. Ayats (Hospital Universitari de Bellvitge, L'Hospitalet de Llobregat, Barcelona); G. Aguilar, R. Badenes, C. Ferrando, D. Navarro, J. A. Carbonell, C. García, A. Gómez, L. Henao, A. Miñana, J. Puig, and F. J. Belda (Hospital Clínico Universitario, Valencia); A. Puppo, J. Garnacho, J. A. Márquez, A. Gutiérrez, and M. Ruiz (Hospital Universitario Virgen del Rocío, Sevilla); V. Jeréz, V. Trasmonte, P. Martínez, D. Pérez, M. Fajardo, C. Gaona, and C. Muñoz, (Hospital Infanta Cristina, Badajoz); G. Tamayo, A. Martínez, F. Labayen, J. R. Iruretagoyena, L. López, and J. L. Hernández (Hospital Universitario Cruces, Barakaldo, Bizkaia); S. Ruiz-Santana, M. A. Hernández, A. Bordes, and N. Ojeda (Hospital Universitario Dr. Negrín, Las Palmas de Gran Canaria); and J. D. Jiménez (Hospital de Don Benito-Villanueva, Don Benito, Badajoz), Spain.

\section{Author details}

${ }^{1}$ Intensive Care Unit, Hospital Universitario de Valme, Universidad de Sevilla, Avenida Bellavista s/n, 41014 Sevilla, Spain. ${ }^{2}$ Intensive Care Unit, Hospital 
Universitario Dr. Negrín, Universidad de Las Palmas de Gran Canaria, Las Palmas de Gran Canaria, Spain. ${ }^{3}$ Mathematics Department, Universidad de las Palmas de Gran Canaria, Las Palmas de Gran Canaria, Spain. ${ }^{4}$ Clinical Unit of Microbiology and Infectious Diseases, Hospital Universitario de Valme, Universidad de Sevilla, Sevilla, Spain. ${ }^{5}$ Intensive Care Unit, Hospital Punta de Europa, Algeciras, Cádiz, Spain. Intensive Care Unit, Hospital Universitario Miguel Servet, Zaragoza, Spain. ${ }^{7}$ Service of Microbiology, Hospital Universitario Miguel Servet, Zaragoza, Spain. ${ }^{8}$ Critical Care Department, Hospital Universitari Joan XXIII, Tarragona, Spain. ${ }^{9}$ Service of Microbiology, Hospital Universitari Joan XXIII, Tarragona, Spain.

\section{Received: 18 February 2016 Accepted: 26 April 2016 Published online: 16 May 2016}

\section{References}

1. León C, Ostrosky-Zeichner L, Schuster M. What's new in the clinical and diagnostic management of invasive candidiasis in critically ill patients. Intensive Care Med. 2014;40:808-9.

2. Lortholary O, Renaudat C, Sitbon K, Madec Y, Denoeud-Ndam L, Wolff L, et al. Worrisome trends in incidence and mortality of candidemia in intensive care units (Paris area, 2002-2010). Intensive Care Med. 2014;40:1303-12.

3. Colombo AL, Guimaraes T, Sukienik T, Pasqualotto AC, Andreotti R, Queiroz-Telles F, et al. Prognostic factors and historical trends in the epidemiology of candidemia in critically ill patients: an analysis of five multicenter studies sequentially conducted over a 9-year period. Intensive Care Med. 2014:40:1489-98.

4. Maubon D, Garnaud C, Calandra T, Sanglard D, Cornet M. Resistance of Candida spp. to antifungal drugs in the ICU: where are we now? Intensive Care Med. 2014;40:1241-55.

5. Shields RK, Nguyen MH, Clancy CJ. Clinical perspectives on echinocandin resistance among Candida species. Curr Opin Infect Dis. 2015;28:514-22.

6. Kullberg BJ, Arendrup MC. Invasive candidiasis. N Engl J Med. 2015;373:1445-6.

7. Clancy CJ, Nguyen $\mathrm{MH}$. Finding the "missing $50 \%$ " of invasive candidiasis: how non-culture diagnostics will improve understanding of disease spectrum and transform patient care. Clin Infect Dis. 2013;56:1284-92.

8. Mikulska M, Furfaro E, Viscoli C. Non-cultural methods for the diagnosis of invasive fungal disease. Expert Rev Anti Infect Ther. 2015;12:103-17.

9. Arvanitis M, Anagnostou T, Fuchs BB, Caliendo AM, Mylonakis E. Molecular and nonmolecular diagnostic methods for invasive fungal infections. Clin Microbiol Rev. 2014;27:490-526.

10. Clancy CL, Nguyen MH. Undiagnosed invasive candidiasis: incorporating non-culture diagnostics in to rational prophylactic and preemptive antifungal strategies. Expert Rev Anti Infect Therap. 2014;12:731-4.

11. León C, Ruiz-Santana S, Saavedra P, Almirante B, Nolla-Salas J. Alvarez- Lerma F, et al. A bedside scoring system ("Candida score") for early antifungal treatment in non-neutropenic critically ill patients with Candida colonization. Crit Care Med. 2006;34:730-7.

12. León C, Ruiz-Santana S, Saavedra P, Galvan B, Blanco A, Castro C, et al. Usefulness of the "Candida score" for discriminating between Candida colonization and invasive candidiasis in non-neutropenic critically ill patients: a prospective multicenter study. Crit Care Med. 2009;37:1624-33.

13. Fortun J, Meije Y, Buitrago MJ, Gago S, Bernal-Martínez L, Pemán J, et al. Clinical validation of a multiplex real-time PCR assay for detection of invasive candidiasis in intensive care unit patients. J Antimicrob Chemother. 2014;69:3134-41.

14. Pittet D, Monod M, Suter PM, Frenk E, Auckentaler R. Candida colonization and subsequent infections in critically ill surgical patients. Ann Surg. 1994;220:751-8.

15. Basseti M, Marchetti M, Chakrabarti A, Colizza S, Garnacho-Montero J, Kett $\mathrm{DH}$, et al. A research agenda on the management of intraabdominal candidiasis: results from a consensus of multinational experts. Intensive Care Med. 2013;39:2092-106.

16. R Development Core Team. A language and environment for statistical computing. Vienna, Austria: Foundation for Statistical Computing. http:// www.r-project.org/.

17. Olaechea-Astigarraga PM. Alvarez- Lerma F, Palomar-Martinez M, Insausti-Ordeñana J, Lopez-Pueyo MJ, Seijas-Betolaza I, et al. Evolución del consumo de antifúngicos en pacientes críticos. Estudio multicéntrico observacional 2006-2010. Enferm Infecc Microbiol Clin. 2012;30:435-40.

18. Azoulay E, Dupont H, Tabah A, Lortholary O, Stahl JP, Francais A, et al. Systemic antifungal therapy in critically ill patients without invasive fungal infection. Crit Care Med. 2012;40:813-22.
19. Ruhnke M. Antifungal stewardship in invasive Candida infections. Clin Microbiol Infect. 2014;20 Suppl 6:11-8.

20. Muñoz P, Valerio M, Antonio Vena A, Bouza E. Antifungal stewardship in daily practice and health economic implications. Mycoses. 2015;58 Suppl 2:14-25.

21. León C, Ruiz-Santana S, Saavedra P, Castro C, Ubeda A, Loza A, et al. Value of $(1 \rightarrow 3) \beta$-D-glucan and Candida albicans germ tube antibody for discriminating between Candida colonization and invasive candidiasis in patients with severe abdominal conditions. Intensive Care Med. 2012;38:1315-25.

22. Martín-Mazuelos E, Loza A, Castro C, Macías D, Zakariya I, Saavedra P, et al. $\beta$-D-glucan and Candida albicans germ tube antibody in ICU patients with invasive candidiasis. Intensive Care Med. 2015;41:1424-32.

23. Ellis M, Al-Ramadi B, Finkelman M, Hedstrom U, Kristensen J, Ali-Zadeh H, et al. Assessment of the clinical utility of serial beta-D-glucan concentrations in patients with persistent neutropenic fever. J Med Microbiol. 2008;57:287-95.

24. Hanson KE, Pfeiffer CD, Lease ED, Balch AH, Zaas AK, Perfect JR, et al. $(1,3)-b-D-G l u c a n$ surveillance with preemptive anidulafungin for invasive candidiasis in intensive care unit patients: a randomized pilot study. PLoS One. 2012;7:e42282.

25. Racil Z, Kocmanova I, Lengerova M, Weinbergerova B, Buresova L, Toskova M, et al. Difficulties in using (1,3)-b-D-glucan as the screening test for the early diagnosis of invasive fungal infections in patients with haematological malignancies-high frequency of false-positive results and their analysis. J Med Microbiol. 2010;59:1016-22.

26. Tissot F, Lamoth F, Hauser PM, Orasch C, Fluckiger U, Siegemund M, et al. Betaglucan antigenemia anticipates diagnosis of blood culture negative intra-abdominal candidiasis. Am J Respir Crit Care Med. 2013;188:1100-9.

27. De Pauw B, Walsh TJ, Donnelly JP, Stevens DA, Edwards JE, Calandra T, et al. Revised definitions of invasive fungal disease from the European Organization for Research and Treatment of Cancer/Invasive Fungal Infections Cooperative Group and the National Institute of Allergy and Infectious Diseases Mycoses Study Group (EORTC/MSG) Consensus Group. Clin Infect Dis. 2008;46:1813-22.

28. Cuenca-Estrella M, Verweij PE, Arendrup MC, Arikan-Akdagli S, Bille J, Donnelly JP, et al. ESCMID guideline for the diagnosis and management of Candida diseases 2012: diagnostic procedures. Clin Microbiol Infect. 2012;18 Suppl 7:9-18.

29. Dellinger RP, Levy MM, Rhodes A, Annane D, Gerlach H, Opal SM, et al. Surviving Sepsis Campaign: international guidelines for management of severe sepsis and septic shock, 2012. Intensive Care Med. 2013;39:165-228.

30. Bailly S, Leroy O, Montravers P, Constantin JM, Dupont H, Guillemot D, et al. Antifungal de-escalation was not associated with adverse outcome in critically ill patients treated for invasive candidiasis: post hoc analyses of the AmarCAND2 study data. Intensive Care Med. 2015:41:1931-40.

31. Martínez-Jiménez MC, Muñoz P, Guinea J, Valerio M, Alonso R, Escribano P, et al. Potential role of Candida albicans germ tube antibody in the diagnosis of deep-seated candidemia. Med Mycol. 2014;52:270-5.

32. Martinez-Jimenez MC, Muñoz P, Valerio M, Alonso R, Martos C, et al. Candida biomarkers in patients with candidaemia and bacteraemia. J Antimicrob Chemother. 2015;70:2354-61.

33. Martínez-Jiménez MC, Muñoz P, Valerio M, Vena A, Guinea J, Bouza E. Combination of Candida biomarkers in patients receiving empirical antifungal therapy in a Spanish tertiary hospital: a potential role in reducing the duration of treatment. J Antimicrob Chemother. 2015;70:3107-15.

34. Mikulska M, Calandra T, Sanguinetti M, Poulain D, Viscoli C, Third European Conference on Infections in Leukemia Group. The use of mannan antigen and anti-mannan antibodies in the diagnosis of invasive candidiasis: recommendations from the Third European Conference on Infections in Leukemia. Crit Care. 2010;14:R222.

35. Chumpitazi BF, Lebeau B, Faure-Cognet O, Hamidfar-Roy R, Timsit JF, Pavese $P$, et al. Characteristic and clinical relevance of Candida mannan test in the diagnosis of probable invasive candidiasis. Med Mycol. 2014;52:462-71.

36. Avni T, Leibovici L, Paul M. PCR diagnosis of invasive candidiasis: systematic review and meta-analysis. J Clin Microbiol. 2011;49:665-70.

37. Nguyen MH, Wissel MC, Shields RK, Salomoni MA, Hao B, Press EG, et al. Performance of Candida real-time polymerase chain reaction, beta-D-glucan assay, and blood cultures in the diagnosis of invasive candidiasis. Clin Infect Dis. 2012;54:1240-8.

38. Held J, Kohlberger I, Rappold E, Grawitz AB, Hackera G. Comparison of (1-3)b-D-glucan, mannan/anti-mannan antibodies, and Cand-Tec Candida antigen as serum biomarkers for candidemia. J Clin Microbiol. 2013;51:1158-64. 